\title{
Integrating Pixel Cluster Indexing, Histogram Intersection And Discrete Wavelet Transform Methods For Color Images Content Based Image Retrieval System
}

\author{
Pijush Kanti Bhattacharjee, Member, IACSIT
}

\begin{abstract}
This paper proposes a new intelligent content based image retrieval (CBIR) system for integrate, disseminate, retrieval, visualize and correlate color images. Three different methods are integrated for searching out the similar images of the query image from a large image databases like consisting of 1798 numbers different images. Then a semantic approach is implemented to minimize the image database i.e. the images are grouped or subdivided. The color images are first converted hue $(H)$, saturation $(S)$, value $(V)$ from $R, G, B$ values, because the HSV model is more authentic than the RGB model. The first method "Pixel Cluster Indexing of the color images" is a completely innovative method. In this, certain range of pixel values say 100-255 either from gray level for monochromatic image or color image in RGB or HSV values are selected and is converted to an equivalent image of the original image i.e. in the equivalent image, the pixel value below than 100 becomes 0 and all other pixels remain same position in the equivalent image as it is in the original image. Hence the equivalent image represents the original image. By this method, the query and the database images are converted to their equivalent images. The original image can be taken in smaller size either by cropping i.e. taking some portion of the image or reducing size by bilinear or nearest neighbor interpolation method for minimizing mathematical complexity. Then the statistical parameters like std, mad of the equivalent images are computed and are combined std and mad in normalized form. Thereafter, this combined value std and mad of the equivalent images derived from the query and database images respectively are compared by Euclidean distance method and the nearest matching images to the query image are ranked accordingly. In the second method, histogram of colors in HSV model is computed for the query as well as the database images respectively. Then this histogram of each $\mathrm{H}, \mathrm{S}$, $\mathrm{V}$ separately between the query and the database images are compared. The minimum numbers of pixel values present in both the comparing histograms is taken for each position and are summed for the whole comparing image sizes.

The total number of pixel values present is normalized and the best matching images for the query image are sorted out from a large image database of $\mathbf{1 7 9 8}$ images.
\end{abstract}

Pijush Kanti Bhattacharjee is an Assistant Professor in the Department of Electronics and Communication Engineering, Bengal Institute of Technology and Management, Santiniketan, P.O. Doranda, West Bengal, Pin-731236, India. He was an Ex Asssitant Director in the Department of Telecommunications (DoT), Government of India, India. He has possessed vast working experience in the field of Telecommunications including Mobile Communications, Image Processing, VLSI etc during last 29 years. He is a member of IACSIT, Singapore; CSTA, USA; IAEng, Hongkong. (phone: +91-33-25954148; email: pijushbhatta_6@hotmail.com).
The third method is Discrete Wavelet Transform (DWT) using Daubechies filter. Since, the approximation co-efficient matrix (CA2) upto two level decomposition of two dimensions (2-D) DWT consists of maximum information of the original image, therefore this approximation co-efficient matrixes are taken for $\mathrm{H}, \mathrm{S}, \mathrm{V}$ values separately for both the query and the database images. Then statistical parameters like mean. Std, correlation co-efficient (corr) are calculated for H,S,V components of approx. co-efficient matrixes separately and these statistical parameters mean, std, corr are combined together in normalized form. The combined value of mean, std, corr in HSV components of the query and the database images are compared by Euclidean distance method and the best matching images are ranked accordingly.

The above three different distinct methods are integrated in normalized form to have the combined effect. The best match similar images are obtained after comparing by Euclidean distance method from the integrated result of Pixel Cluster Indexing, Histogram Intersection and DWT revealing the query and the database images. This innovative integrated process shows the best reliable performance in CBIR system.

Keywords-Pixel Cluster Indexing, Histogram Intersection, Discrete Wavelet Transform, image cropping, bilinear interpolation, nearest neighbor interpolation., mean, std, mad, corr, image matching. image semantics.

\section{INTRODUCTION}

With the increasing techniques in Image Processing and popularization of the internet, Content Based Image Retrieval (CBIR) has developed a new region for identifying the best match images from an image database. A new generation of intelligent data base system has been evolved for integrate, disseminate, retrieval, visualize and correlate images [1]-[10]. There is study that deals with the choice of features for most problem of interest. We are finding characteristic of the distance measure that is relatively independent of the choice of the feature space. Researchers in CBIR identify low level features color, texture, shape, statistical parameters etc and high level features like semantics, fuzzy logic etc for interactive image understanding. Also the large computational and storage capacity is necessary for visual databases. For this, it becomes necessary to build an automatic and efficient retrieval system to browse through the entire database. Image retrieval does not entail solving the general image understanding problem. It may be sufficient that a retrieval 
system present similar images, similar in some user defined sense. Different statistical and mathematical applications are adopted to minimize the features of the data base images and the query image and then comparing the above features by different distance method like city block or Manhattan distance, Euclidean distance etc.

Image Database

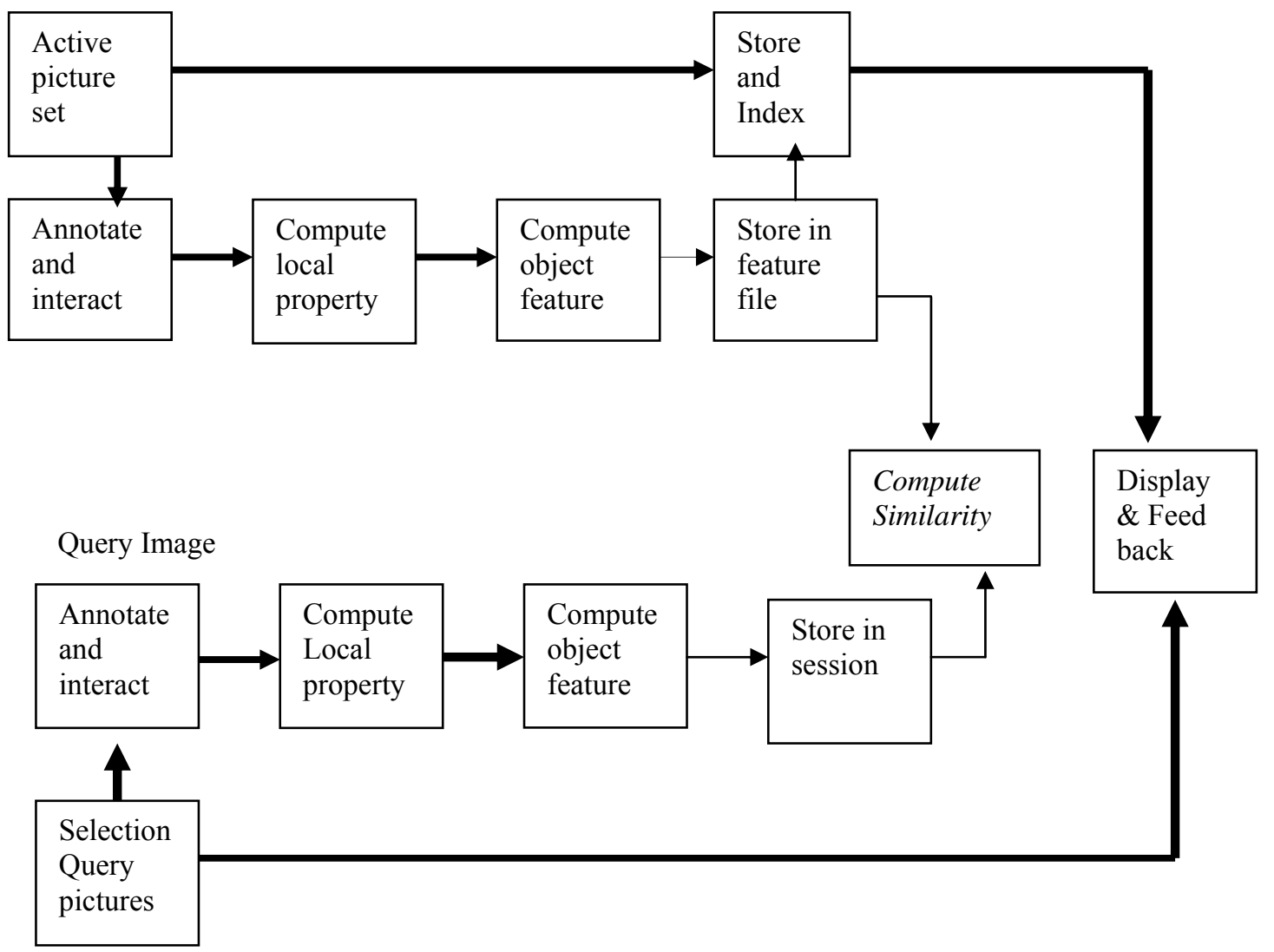

Fig.1. Basic block diagram of the similar images search for the query image from image database system.

$\longrightarrow$

Feature vector

Image field
This approach relates with the image features that imply visual clues such as color, texture with different mathematical and statistical features like mean, std, mad, corr etc. Previous approaches mainly concentrate on extracting single feature, but this technique integrates all three distinct type of features i.e. combined statistical parameters std, mad of pixel cluster indexing, combining histogram of color intersection hue-saturation-value components and statistical parameters like mean, std, corr of approximation coefficients (CA2) from discrete wavelet transform (DWT).

The input images are processed to extract features which are stored along with the images in the database. When a query image is placed, it is similarly processed to extract its features which are then matched with the feature vectors present in the database. A ranked set of images with high matching scores are presented as the retrieval output.

In the present state of the art [1]-[10] the automatic aspect of indexing, which is an important advantage of visual information systems, supports weak knowledge description and therefore, weak very results. The basic idea of image retrieval by image example is to extract the characteristic features from target images which are then matched or compared with that of the query image. These features are typically derived from shape, texture, color properties or statistical attributes of the query and the target images. After matching, the images are ordered with respect to the query image according to their similarity measure and are displayed for viewing [9]-[16].

The similarity measure is based on the distance between the feature vectors of images in the feature space, with 1.0 representing the highest similarity and 0.0 representing no similarity. The measurement is determined by computing the features of texture, color, shape and statistical parameters. Given two images $\mathrm{A}$ and $\mathrm{B}$, and $0.0 \leq \operatorname{sim}(\mathrm{i}, \mathrm{j}) \leq$ 1.0. The effectiveness of this measure is capturing visual similarity between database images and templates can be demonstrated by showing the existence of different similarity ranges for distinct clusters.

\section{COMPUTATIONAL PART}

Different mathematical formulae and concepts are used in this section. 


\section{A.RGB image is converted to HSV image}

R (Red), G (Green), B (Blue) parts of the image are converted to HSV (Hue, Saturation, Value), because HSV Color representation assures like human color perception. Hue $(\mathrm{H})$ is the color content in the image, Saturation (S) identifies relative white content of a color, Value (V) or Intensity (I) indicates a light or surface color from dim to bright.

$$
\begin{gathered}
\mathrm{V}=\frac{\mathrm{R}+\mathrm{G}+\mathrm{B}}{3} \\
\mathrm{~S}=1-\frac{3}{\mathrm{R}+\mathrm{G}+\mathrm{B}}[\min (\mathrm{R}, \mathrm{G}, \mathrm{B})] \\
\mathrm{H}=\mathrm{Cos}^{-1}\left\{\frac{\frac{1}{2}[(\mathrm{R}-\mathrm{G})-(\mathrm{R}-\mathrm{B})]}{\left[(\mathrm{R}-\mathrm{G})^{2}+(\mathrm{R}-\mathrm{B})(\mathrm{G}-\mathrm{B})\right]^{1 / 2}}\right\}
\end{gathered}
$$

$\mathrm{H}=360^{\circ}-\mathrm{H}$ if $\mathrm{B}>\mathrm{G}$. Since $\mathrm{H}$ lies between 0 to $180^{\circ}$.

Therefore, Hue and Saturation part determine the color image characteristics.

\section{B.Pixel Cluster Indexing Method}

Some of the pixels range (say 100 to 255 taken for gray level or HSV level where HSV level normalized upto 255) is selected as lying in the corresponding position of the image and other pixel level (say 0-100) are taken as zero i.e. finding or indexing pixel 100 to 255 only and taking as it is in the equivalent image with rest of the pixel values $(<100)$ taken as zero. By this, the number of pixels is becoming less in the equivalent or corresponding image of the original image and it reveals less computational complexity.

\section{C.Statistical Parameters}

1) Mean, $\mu=\frac{1}{\mathrm{MxN}} \sum_{\mathrm{x}=1}^{\mathrm{M}} \sum_{\mathrm{y}=1}^{\mathrm{N}} \mathrm{f}(\mathrm{x}, \mathrm{y})$, Where $\mathrm{MxN}$ is the size of the image $f(x, y)$.

2) Standard Deviation,

$$
\sigma=\sqrt{\frac{1}{\mathrm{MxN}} \sum_{\mathrm{x}=1}^{\mathrm{M}} \sum_{\mathrm{y}=1}^{\mathrm{N}}[\mathrm{f}(\mathrm{x}, \mathrm{y})-\mu]^{2}}
$$

3) Mean Absolute Deviation,

$$
\mathrm{MAD}=\frac{1}{\mathrm{MxN}} \sum_{\mathrm{x}=1}^{\mathrm{M}} \sum_{\mathrm{y}=1}^{\mathrm{N}} \mid \mathrm{f}(\mathrm{x}, \mathrm{y})-\text { median } \mid
$$

4) Correlation Coefficient: Correlation is the matching between two image matrices. If $\mathrm{W}(\mathrm{x}, \mathrm{y})$ of size JxK be an image is corelated with another image $f(x, y)$ of size $\mathrm{MxN}$, where $\mathrm{M} \geq \mathrm{J}$ and $\mathrm{N} \geq \mathrm{K}$.

Correlation coefficient,

$$
R(m, n)=\frac{\sum_{x=1}^{M} \sum_{y=1}^{N} f(x, y) w(x-m, y-n)}{\left[\sum_{x=1}^{M} \sum_{y=1}^{N} f^{2}(x, y)\right]^{1 / 2}}
$$

Generally correlation coefficients between two images matrices having same size are calculated and sorted according descending order i.e. largest correlation coefficient value is the nearest or the best match.

\section{D.Histogram Intersection Method}

Histogram Intersection $=\sum_{\mathrm{j}=1}^{\mathrm{n}} \min \left(\mathrm{I}_{\mathrm{j}}, \mathrm{M}_{\mathrm{j}}\right)$

Where $\mathrm{I}$ is the query image and $\mathrm{M}$ is the model or the data base image, each consisting of $\mathrm{n}$ bins in the histogram,

Normalized Histogram Intersection,

$$
H(I, M)=\frac{\sum_{j=1}^{n} \min \left(I_{j}, M_{j}\right)}{\sum_{j=1}^{n} M_{j}}
$$

Again, Sum of Absolute Differences between respective bins of the query and the data base image is $\sum_{j=1}^{n}\left|I_{j}-M_{j}\right|$, It is shown in [22],

$$
\begin{array}{r}
\text { where } \sum_{j=1}^{n} I_{j}=\sum_{j=1}^{n} M_{j}, \\
1-H(I, M)=\frac{\sum_{j=1}^{n}\left|I_{j}-M_{j}\right|}{2 * \sum_{j=1}^{n} M_{j}}
\end{array}
$$

Histogram Intersection is related with sum of absolute differences between respective bins of $\mathrm{H}$ (hue), $\mathrm{S}$ (saturation), V (value).

\section{E.Discrete Wavelets Transform Method}

The translated and dilated wavelets derived from the mother wavelet are given by,

$$
\begin{aligned}
& \psi_{m n}(k)=2^{\frac{-m}{2}} \psi\left(2^{-m} k-n\right) \\
& x(k)=\sum_{m, n} w(m, n) \psi_{m n}(k)
\end{aligned}
$$

where $m, n, k$ are some integers. The wavelet decomposition for the signal $x(k)$ is obtained as follows: where

$$
w(m, n)=\left\langle x, \psi_{m n}\right\rangle \equiv \sum_{k} x(k) \psi_{m n}(k)
$$

are called the wavelet coefficients. The Approximation Coefficient Matrix (CA2) i.e. Low-Low Component of two level Wavelet Decomposition consists of maximum information of the signal, Hence it is considered to extract different kinds of features for CBIR. Here we are applying Daubechies wavelet transform.

\section{F.Distance Functions}

Minkowski form of general distance measure between $\mathrm{H}_{0}$ and $\mathrm{H}_{1}$ is

$$
\mathrm{D}\left(\mathrm{H}_{0} \mathrm{H}_{1}\right)=\left[\left|\mathrm{H}_{0}(\mathrm{i})-\mathrm{H}_{1}(\mathrm{i})\right|^{\mathrm{p}}\right]^{1 / \mathrm{p}}
$$


1) For $\mathrm{p}=0$, It gives City Block or Manhattan distance. City Block Distance between two coordinates $(\mathrm{i}, \mathrm{j})$ and $(\mathrm{h}, \mathrm{k})$ is defined by $\mathrm{D}[(\mathrm{i}, \mathrm{j}),(\mathrm{h}, \mathrm{k})]=|\mathrm{i}-\mathrm{h}|+$ $|\mathrm{j}-\mathrm{k}|$

Manhattan distance between two pixels of two different images $\mathrm{x}$ and $\mathrm{y}$ is

$\mathrm{D}=[\mathrm{x}(\mathrm{i}, \mathrm{j})-\mathrm{y}(\mathrm{i}, \mathrm{j})]$

2) If $\mathrm{p}=2$, It gives Euclidean distance $\mathrm{D}[(\mathrm{i}, \mathrm{j}),(\mathrm{h}, \mathrm{k})]=$ $\sqrt{(\mathrm{i}-\mathrm{h})^{2}+(\mathrm{j}-\mathrm{k})^{2}}$

In case of pixels matching lying on the same co-ordinates between the two images, Manhattan distance becomes equal to Euclidean distance.

\section{G.Retrieval Efficiency}

1) Retrieval Efficiency or Number of Pixels between two images are exactly matching:

2) Retrieval Efficiency $(\eta)=\frac{P}{M X N} X 100 \%$

Where $\mathrm{P}$ is the number of gray value in monochromatic image and $\mathrm{R}, \mathrm{G}, \mathrm{B}$ value or $\mathrm{H}, \mathrm{S}, \mathrm{V}$ value in color image similar [ i.e. $f_{q}(x, y)-f_{d}(x, y)=0$ ] between the query image and the data base image, $\mathrm{M} \mathrm{X} \mathrm{N}$ are the total number of pixels in an image.

\section{Methodology}

The five type of image matching is incurred in this paper.

3) Pixel Cluster Indexing and statistical parameters like std and mad of equivalent images are compared.

4) Taking Histogram of Colors in HSV model and applying histogram intersection method [22].

5) Approximation Co-efficient matrix of DWT (Discrete Wavelet Transform) and compare by combining its statistical parameters mean, std, corr.

6) Integrating Histogram Intersection and Discrete Wavelet Transform methods.

7) Integrating Pixel Cluster Indexing, Histogram Intersection and Discrete Wavelet Transform methods.

\section{A. Pixel Cluster Indexing Method}

HSV components of the query and the database images are normalized or fixed between 0-255. Then it is indexed for a particular range of pixel values, here pixels 100 to 255 are taken as it is and pixels 0 to 99 are taken as 0 , since we may consider pixel value less than 100 as 0 . In case of gray values, less than 100 may be considered as completely dark. Thus, the indexed image becomes the equivalent image for a particular image. Thereafter, statistical parameters std and mad of the indexed images are computed for the query image and the database images respectively and the two parameters std and mad are combined together in normalized form. The combined std and mad values of the query and database images are compared or matched by Euclidean distance method. The best fifteen similar matching images from the database from 1798 images are ranked as shown in fig. 2. The original image may be taken in smaller size either by cropping i.e. taking some portion of the image or reducing size by bilinear or nearest neighbor interpolation method for moderate matching.
It is a completely innovating method which can be implemented to any type of images. In this only some of sample pixels are taken. This process minimizes time and mathematical complexity. Ultimately it yields a very soft and easy computing technique for image matching in content based image retrieval.

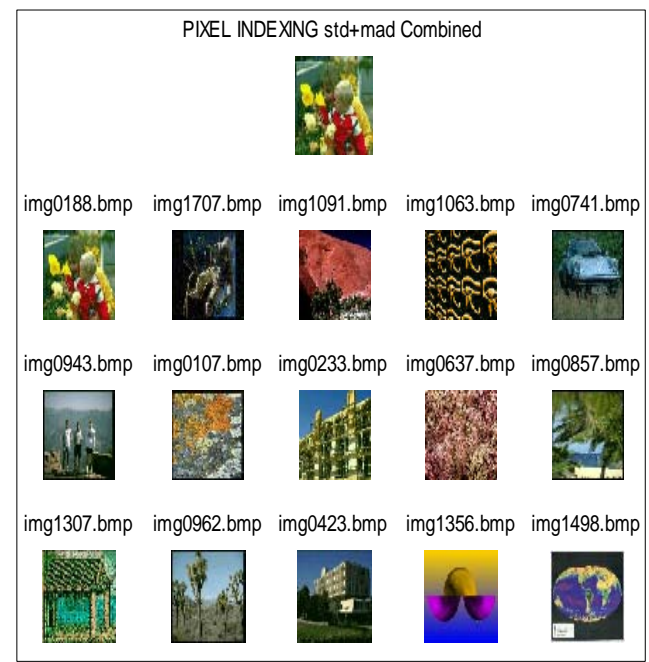

Fig. 2. Retrieved top fifteen similar images from the database of 1798 images by Pixel Indexing technique

\section{B. Histogram Intersection Method}

Histogram i.e. number of pixels having gray or other values like $H, S, V$ components are taken for the data base and the query images [22]. Then minimum number of corresponding pixels according to histogram of the query and database images is found out and normalized the matching value. The matching images are sorted from the database of 1798 images as shown in fig. 3.

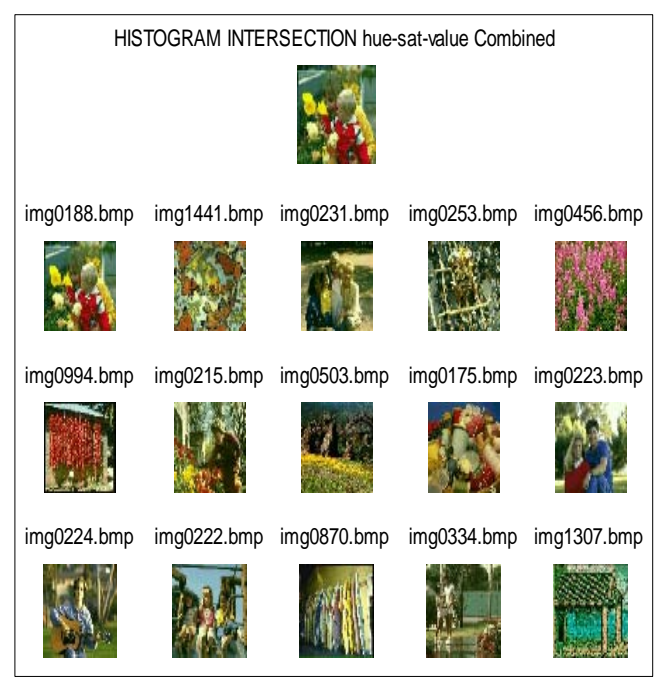

Fig. 3. Retrieved top fifteen similar images from the database of 1798 images by Histogram Intersection technique

\section{Discrete Wavelet Transform (DWT) Method}

Wavelets are based on multiresolution theory i.e. representation and analysis of images at more than one 
resolution [1]-[3], [23]. The features that might go undetected at one resolution may be easy to locate at another. Important techniques of multiresolution analysis are subband coding. In subband coding, an image is decomposed into a set of band limited components, called subbands, which can be reassembled to reconstruct the original image without error. Since the filter outputs after down sampling in two stages are called the approximation, vertical detail, horizontal detail and diagonal detail subbands or components of the image respectively. One or more of these subbands can be split into four smaller subbands, which can be split again and so on. Each stage subband age size is reduced to half of the original image. We applied Daubechies filter. In 2-D Discrete Wavelet Transform using Daubechies filter of an image, having ability to repeatedly decompose an image in the low frequency components or channels. Since, the approximation coefficient matrix or low-low component (CA2) up to two levels decomposition of 2-D DWT consists of maximum information of the original image, we take the approx. coefficient matrix (CA2) of the query and the data base images and calculate mean, std, correlation coefficient of the approx. coefficient component of 2-D DWT, then combine or integrate the feature parameters like mean, std, corr coefficients. Matching similar images from the database images is done

by comparing the combined parameters of the query image with that of the data base images with Euclidean distance method and are ranked accordingly. The result is shown in fig. 4 by retrieving from large image databases of 1798 images.

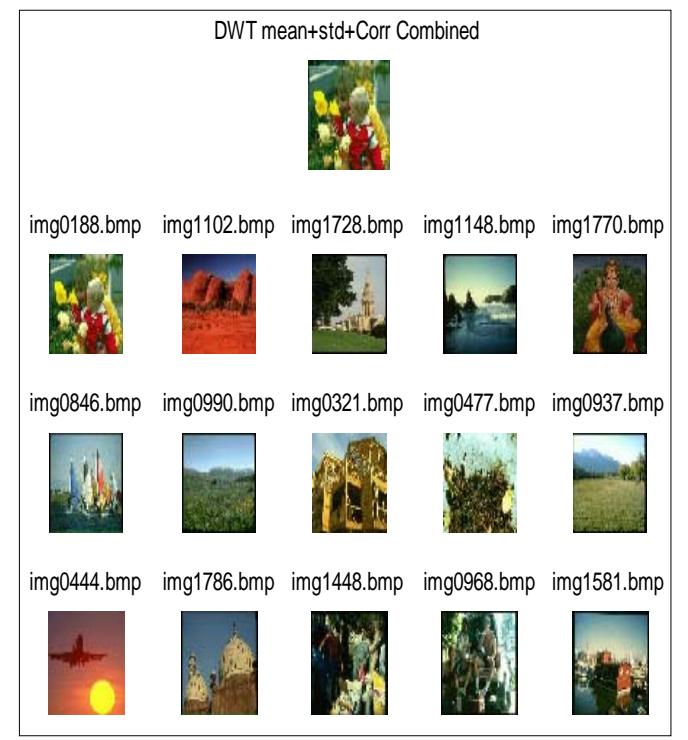

Fig. 4. Retrieved top fifteen similar images from the database of 1798 images by Discrete Wavelet Transform technique

D. Integrating Histogram Intersection and Discrete Wavelet Transform Methods

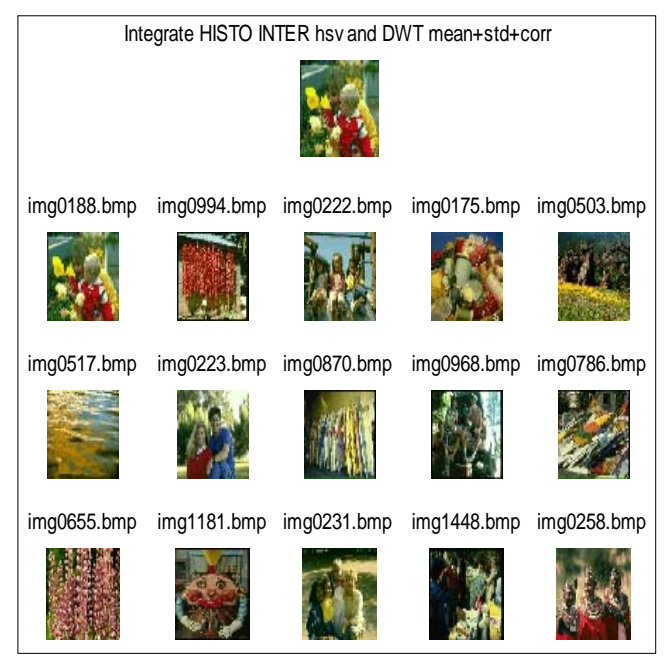

Fg. 5. Retrieved top fifteen similar images from the database of 1798 images by Integrating Histogram Intersection and DWT technique

These two methods are integrated by adding the two distinct features in normalized form and then comparing the combined result of the query image to that of the database images by Euclidean distance method. The ultimate matching images are ranked accordingly as shown in fig. 5.

\section{E. Integrating Pixel Cluster Indexing, Histogram Intersection and Discrete Wavelet Transform Methods}

Thereafter we integrate all the above three distinct methods by summing up the three distinct features in normalized form. Then combined effect feature vectors of the query and the data base images are compared by Euclidean distance method. At last the integrating effect of all the three different processes is obtained.

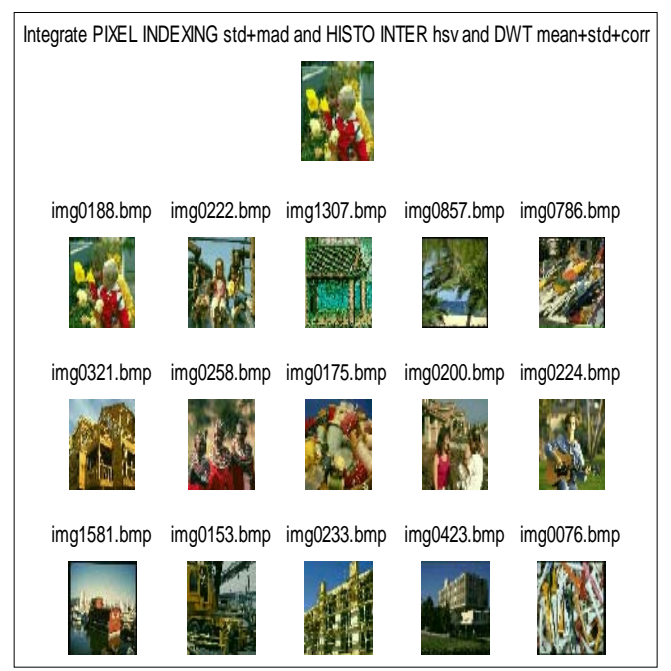

Fig. 6. Retrieved top fifteen similar images from the database of 1798 images by Integrating Pixel Cluster Indexing, Histogram Intersection and Discrete Wavelet Transform technique

This method is first time invented in content based image retrieval. It is practically shown in fig. 6. In Fig. 7, it compares the completely matching pixel values between the query image and the first fifteen similar images revealing 
from integrating of Pixel Indexing, Histogram Intersection and DWT technique with Histogram Intersection and DWT technique with Pixel Cluster Indexing technique only. By comparing the retrieval efficiencies of these techniques, we get an overall knowledge about the perfection of the techniques i.e. efficiency of the applied methods. Also the query image is taken as a texture image in Fig. 8 and the corresponding matching similar images from the database images (1798 images) are ranked accordingly. Using Pentium-4 personal computer with MATLAB-7 version software, the whole process for simulation of the CBIR program for searching similar images of the query image from 1798 number of the database images takes $173.688 \mathrm{sec}$ time only. If we can grouped or subdivided the total database images and each group is having smaller number of images than that of the total database images, then the searching time for finding out the similar images of the query image will be obviously very less. This is achieved by distributing the total number of database images into smaller groups by the semantic approach. By applying the semantic approach, not only the computer memory locations require very less i.e. within a stipulated memory capacity, but a general idea of the similar images are also made searching for the query image. The searching job is more signified in a proper direction.

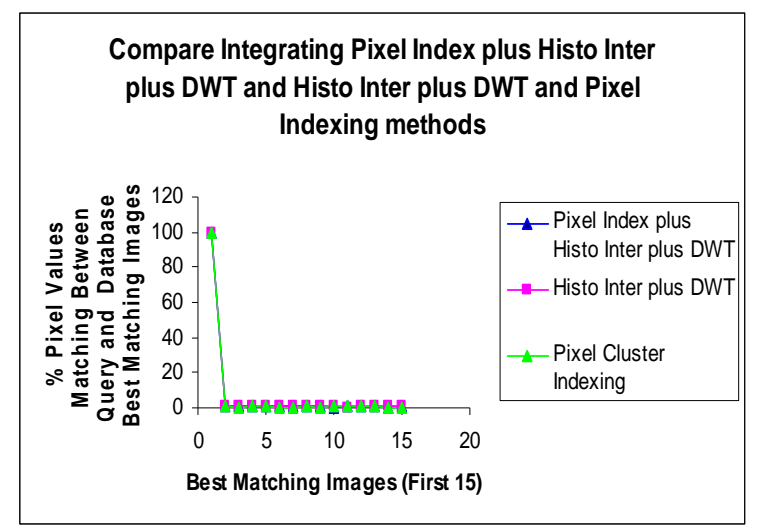

Fig. 7. Compare between Integrating Pixel Indexing plus Histogram Intersection plus DWT technique with Integrating Histogram Intersection plus DWT technique with Pixel Cluster Indexing technique.

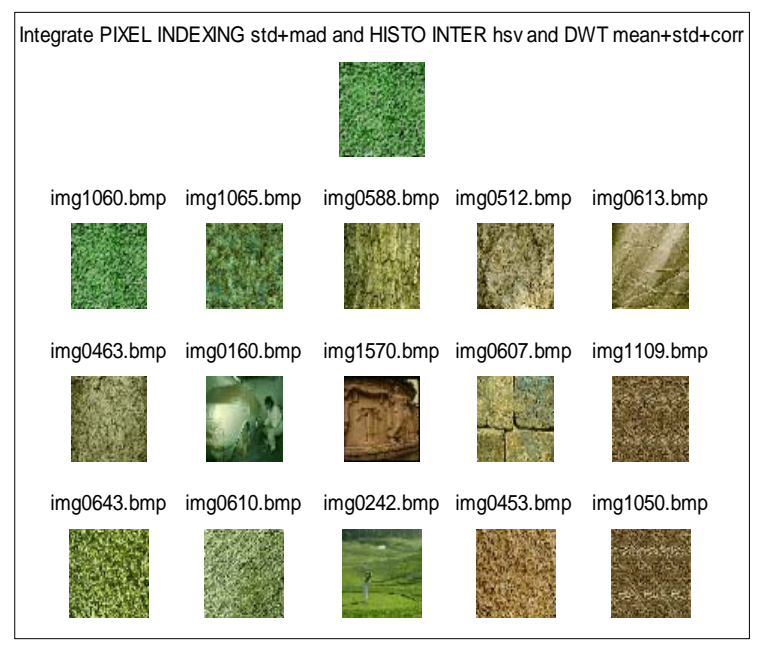

Fig. 8. Retrieved top fifteen similar images from the database of 1798 images by Integrating Pixel Cluster Indexing, Histogram Intersection and
Discrete Wavelet Transform technique, query image taken as Texture Image.

\section{SEMANTICS}

Queries based on content of the image features (e.g. color, texture etc) are not powerful enough to specify knowledge queries based on semantics, because the associations between the content and the semantic in the user's mind are weakly declared [17]-[21]. The user has to define the semantic i.e. some reasons he is looking for in terms of visual description (e.g. houses, lakes, mango etc). In the query, the similarity between feature vectors may be useful, but they are not the ultimum. Techniques for retrieval by image semantics are much less developed and also much more difficult problem. Despite some success like automatic scene classification [18], learning semantic association's small images or particular pattern [17] is described.

By grouping the total images databases in several subgroups according to image semantics, we can have very less number of images in a subgroup and the time require to sort out the similar images to the query image is also very less or minimum. This challenge begins a new generation of information retrieval systems in which the knowledge is extracted automatically and is well positioned to drive the retrieval process accurately. In semantic approach, a hierarchical image [20] classification is done. Although many visual information systems have been developed recently [4], [7], [21], none of these systems operate by considering knowledge extracted from image repositories. In this paper, first I divide the whole image databases into small number of subgroups in accordance with the image semantics i.e. some sort of similar pattern or images identifying by human eye. This subgroups can be named as Image-I, Image-II, Image-III,..... so on. All type of images is falling into these ten image categories or subgroups.

Semantics matching by human eye in following subgroups.

(i) House or Construction ........... IMAGE-I
(ii) People or Man/Woman/Child......IMAGE-II
(iii)WaterReservoir, lake, $\quad$ Ponds,

(iii)

Sea,

(iv) Animal and Birds...................IMAGE-IV

(v) Plants, Trees and Flowers............ IMAGE-V

(vi) Instrument or Machine............... IMAGE-VI

(vii) Transportation, Vehicles, Train, Sheep, Aero plane............................ IMAGE-VII

(viii)Food, Fruits and Vegetables …............................................... IMAGE-

VIII

(ix)Texture and Background Images ....................................................IMAGE-IX

(x)Others........................................ IMAGE-X

First of all the query image is matched to which subgroup it belongs. Then we combine the low level features like Pixel Cluster Indexing std and mad statistical parameters, histogram of colors intersection and approximation coefficients of DWT (Discrete Wavelet Transform) comparing mean, std, corr parameters The normalized combined parameter of the query image is compared for searching similar images with that of the database images by Euclidean distance method, as shown in Fig. 9. For example, only 394 images belong to category IMAGE-VII consisting of all type vehicles in an image database 
consisting of 1798 images. The whole process of searching out similar images for the query image from the newly database consisting of 394 images under IMAGE-VII takes $75.203 \mathrm{sec}$ time by Pentium-4 PC and MATLAB-7 version software, whereas to search from the database of 1798 images, it takes $173.692 \mathrm{sec}$ time. Therefore, the application of this semantic approach gives the fastest solution in a CBIR system.

This semantic approach develops a new technique which can minimize time to search out the exact image from a limited database images. Thus it can make short and easy implementations in CBIR for a large image database by cutting in several small databases. In fine, all databases are joined together to form the original database containing large or huge number of images in all patterns like natural, artificial, texture, animation images etc. Therefore this semantic approach delivers a new direction and a beautiful scheme in CBIR, but to arrange the images in a particular type or range, it requires some careful and hard some work; mainly it is done by human approach. If this human interaction can be shifted by soft computing technique, it is a very nice method above all.

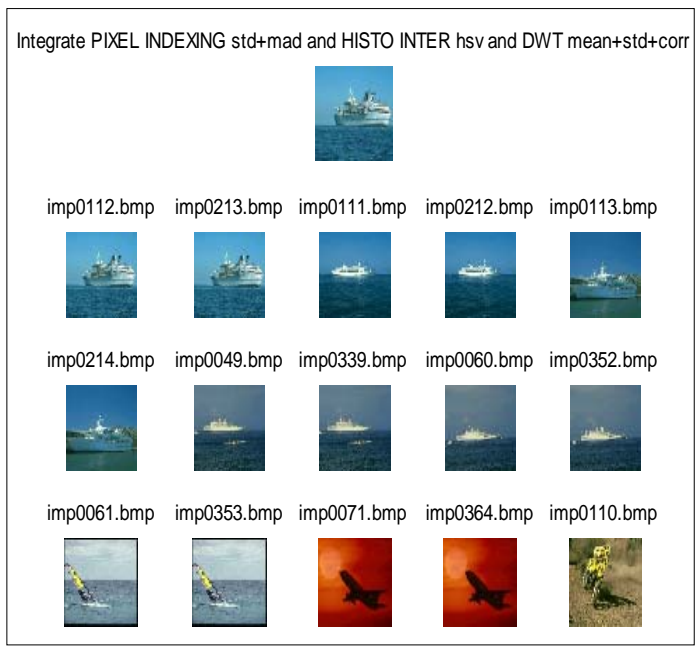

Fig. 9. Retrieved top fifteen similar images from the database of 394 images under IMAGE-VII by Integrating Pixel Cluster Indexing, Histogram Intersection and Discrete Wavelet Transform technique

\section{Advantages Of The Proposed Integrating TECHNIQUE IN CBIR SYSTEM}

This integrating method has several advantages which are mentioned below:

1) This technique is integrating different independent methods such as integrating pixel cluster indexing, histogram intersection and discrete wavelet transform methods. Thus it is a unique one in the field of Content Based Image Retrieval (CBIR) system.

2) The results or the best matching images derived by this technique are very much accurate and reliable comparing to the other existing methods in CBIR. Therefore efficiency of this process is highly improved one.

3) This three independent integrating methods are summing up the merits of each individual system and likely it ensures the best match images to the query image from a large image database.

4) Since all the attributes of the images are computed by mathematically and statistically and accordingly decision is taken, so there is no chance for mistake or wrong choice for the best match images to the query image.

5) Pentium-V or higher order processor is able to assure the final output i.e. to find out best matching images nearer to the query image from large image database within a real time basis e.g. Pentium- $V$ processor with Matlab-7 version software, the best match images are displayed or printed within $173.688 \mathrm{sec}$ from the image database consisting of 1798 images.

6) This integrating technique is highly precision and suitable one in any field of CBIR system like missile controlling (guiding), medical image processing, planets scanning etc.

\section{CONCLUSION}

The proposed integrating technique of Pixel Cluster Indexing, Histogram Intersection and Discrete Wavelet Transform with the image subdivided by the semantic approach is the best suitable method for content based image retrieval from a large image database. This is applied to all sorts of images like natural, texture, animation etc. This CBIR system comprises with integrating three separate methods and application of the semantic approach. Therefore, this process yields the best result within a stipulated real time.

\section{REFERENCES}

[1] A. K. Jain, Fundamental of Digital Image Processing, Englewood cliffs, N.J:Prentice Hall,1989.

[2] R. Jain, R. Kasturi and B. Shunk, Machine Vision, McGraw-Hill, 1995.

[3] A. W. M. Smeulders, S. Santini, A. K. Jain, A. Vailya, A. Gupta and R. Jain, "Content Based Image Retrieval at the End of Early Years," IEE Trans PAMI, vol 22, No. 12, pp 1349-1380 Dec 2000.

[4] C. Faloutsos, M. Flickner, W. Niblack, D. Petrovic, W. Equitz and R. Barber, "Efficient and Effective Query by Image Content," Research Report, IBM Alameda Research Center, 1993.

[5] A. Gupta and R. Jain, "Visual Information Retrieval," Comm. ACM, vol. 40, no.5, May 1997.

[6] R. Jain, "Content-Based Multimedia Information Management," Proc Int'l Conf. Data Engg (ICDE), pp252-253,1998.

[7] A. Pentland, R. W. Picard and S. Sclaroff, "Photobook: Tools for Content-Based Manipulation of Image Databases," Proc. SPIE-94, pp.34-47, 1994.

[8] W. W. Chu, C. C. Hsu, A. F. Cardenas and R. K. Taira, "KnowledgeBased Image Retrieval with Spatial and Temporal Constructs," IEEE Transctions knowledge \& Data Engineering, vol.10, no.6, pp 872-888, 1998.

[9] T. Gevers and A. W. M. Smeulders, "PictoSeek: A Content-based Image Search Engine for the World Wide Web", in Proc. Visual Information Systems, San Diego, CA, 1997, pp.93-100.

[10] W. Grosky and R. Mehrotra, "Special Issue on Image Data Base Management," Computer, vol.22, no.12, Sept, 1989.

[11] IFIP, Visual Database Systems I and II, Amsterdam, The Netherlands Elsevier, 1989 and 1992.

[12] W. Niblack and R. Jain, Eds., Proc. Storage and Retrieval for Image and Video Databases I,II and III, Bellingham, WA:SPIE, 1993,1994 and 1995, vol.1,908;2,185; and 2,420.

[13] A. Califano and R. Mohan, "Multidimensional Indexing for Recognizing Visual Shapes," IEEE Trans. Pattern Analysis \& Machine Intelligence., vol 16, pp.373-392, Apr 1994.

[14] J Sklansky, "Image Segmentation and Feature Extraction," IEEE Trans on Systems, Man and Cybernetics, vol8, pp237-247, 1978. 
[15] W. Y. Ma \& B. S. Manjunath, "Texture-based Pattern Retrieval for Image Databases," Multimedia Tools and Applications 1(2), pp 35-51, 1996.

[16] N. G. Kingsbury, "The Dual-Tree Complex Wavelet Transform: A New Efficient Tool for Image Restoration and Enhancement," Proc European Signal Processing Conf. pp319-322, Sept 1998.

[17] Chabne Djerabe, "Association and Content-Based Retrieval", IEEE transaction on Knowledge and Data Engineering, vol.15, No.1, JanFeb2003.

[18] Oliva A \& Torriba AB, "Global Semantic Classification of Scenes Using Power Spectrum Templates," CIR-99, the Challenge of Image Retrieval, New Castle, Feb 99.

[19] Chang S F, Chen W \& Sundaram H, "Semantic Visual Templates: Linking Visual Features to Semantic", IEEE Int Conf on Image Processing (ICIP1998), Chicago, Illinois, pp531-553, 1998.

[20] J. Huang, R. Kumar and R. Zabih, "An Automatic Hierarchical Image Classification Scheme," Proc. Sixth ACM Int'l Conf. Multimedia (ACM MM-98), 1998.

[21] V. Raghavan, G. Jung, and P. Bollman, "A Critical Investigation of Recall and Precision as Measures," ACM Trans. Information Systems, vol.7, no.3, pp205-229, July89.

[22] M. J. Swain and D. H. Ballard, "Color Indexing", International Journal of Computer Vision, vol.7, no.1, pp.11-12, 1991.

[23] S.G. Mallat, "Multifrequency Channel Decompositions of Images and Wavelet Models," IEEE Trans. Acq. Speech Signal Processing, vol.37, pp2091-2110, 1989 .

Author: Dr. Pijush Kanti Bhattacharjee is a pioneer in Engineering,

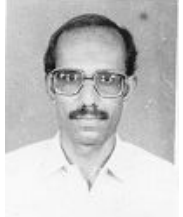

Management, Law, Indo-Allopathy, Herbal, Homeopathic and Yogic medicines. He is having qualifications M.E, MBA, MDCTech, A.M.I.E, B.Sc, B.A, LLB, BIASM, CMS, PET, EDT, FWT, DATHRY, B.Mus, KOVID, DH, ACE, FDCI etc. He worked Department of Telecommunications (DoT), Govt. of India from June 1981 to Jan 2007 (26 years), lastly holding Assistant Director post at RTEC [ER], DoT,

Kolkata, India. Thereafter, he worked at IMPS College of Engineering and Technology, Malda, WB, India as an Assistant Professor in Electronics and Communication Engineering Department from Jan,2007 to Feb,2008 and Feb, 2008 to Dec, 2008 at Haldia Institute of Technology, Haldia, WB India. In Dec, 2008 he joined at Bengal Institute of Technology and Management, Santiniketan, WB, India in the same post and department. He has written two books "Telecommunications India" \& "Computer". He is a member of IACSIT, Singapore; CSTA, USA; IAEng, Hongkong and IE, India. 